\title{
ON THE HAUPTVERMUTUNG FOR MANIFOLDS
}

\author{
BY D. SULLIVAN
}

Communicated by William Browder, January 30, 1967

The "Hauptvermutung" is the conjecture that homeomorphic (finite) simplicial complexes have isomorphic subdivisions, i.e. homeomorphic implies piecewise linearly homeomorphic. It was formulated in the first decade of this century and seems to have been inspired by the question of the topological invariance of the Betti and torsion numbers of a finite simplicial complex.

The Hauptvermutung is known to be true for simplicial complexes of dimension $<4,{ }^{1}$ but there are counterexamples in each dimension $>4$ (Milnor, 1961).

The Milnor examples, $K$ and $L$, have two notable properties:

(i) $K$ and $L$ are not manifolds,

(ii) $K$ and $L$ are not locally isomorphic.

Thus it is natural to restrict the Hauptvermutung to the class of piecewise linear $n$-manifolds, simplicial complexes where each point has a neighborhood which is piecewise linearly homeomorphic to Euclidean space $R^{n}$ or Euclidean half space $R_{+}^{n}$.

We assume that $H_{3}(M, Z)$ has no 2-torsion. ${ }^{2}$

The Main Theorem. Let $h$ be a homeomorphism between compact $P L$-manifolds $L$ and $M$. Then for some integer $p$

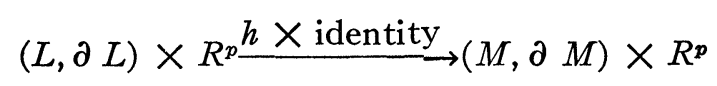

is properly homotopic to a PL-homeomorphism. If $\operatorname{dim} M \geqq 6$ and $\pi_{1} M=\pi_{1} \partial M=0$, then $h$ is homotopic to a PL-homeomorphism.

There are three steps in the proof of the Main Theorem.

For simplicity we assume now that $M$ and $L$ are closed simply connected PL-manifolds of $\operatorname{dim}>4$.

Definition 1. Let $g: L \rightarrow M$ be a homotopy equivalence. A triangulation of $g$ is a homotopy of $g$ to a $P L$-homeomorphism.

11-complexes, obvious. 2-manifolds-Rado, 1926. 2-complexes-Papakyriakopoulos, 1943. 3-manifolds-Moise, 1953. 3-complexes-E. Brown, 1964.

${ }^{2}$ We state this condition in terms of $H_{3}$ instead of $H^{4}$ to suggest a connection with the three dimensional Poincare conjecture. 
The obstruction theory. Let $g: L \rightarrow M$ be a homotopy equivalence. Then there is an obstruction theory for constructing a triangulation of $g$. The obstructions are determined geometrically in terms of partial triangulations of $g$ defined on thickened skeletons of $L$. They may also be interpreted as the homotopy obstructions for deforming a map of $L$ into a universal space $F / P L$ to a constant. The obstructions lie in

$$
H^{4 i}(L, Z) \quad \text { or } \quad H^{4 i+2}\left(L, Z_{2}\right), \quad 0<4 i, \quad 4 i+2<\operatorname{dim} L .
$$

The universal space $F / P L$ is defined so that the set of homotopy classes of maps of a finite complex $X$ into $F / P L$ are in one to one correspondence with the set of stable equivalence classes of $F / P L$ bundles over $X$. An $F / P L$-bundle over $X$ is a piecewise linear $R^{n}$ bundle over $X, p: E \rightarrow X$, together with a fibre homotopy equivalence $t: E \rightarrow X \times R^{n}$, i.e. $p_{1} t=p$ and $t$ is a proper homotopy equivalence on each fibre. $(E, t) \sim 0$ iff $t$ is homotopic through fibre homotopy equivalences to a piecewise linear bundle equivalence.

If $W$ is an oriented manifold and $\partial W=n L$, then we call the space $M$ obtained from $W$ by identifying the various copies of $L$ to one another a $Z_{n}$-manifold, and we set $\delta M=L$. A variety is a disjoint union of $Z_{n}$-manifolds of various dimensions for various $n$ 's.

Definition 2. If $\eta=(E, t)$ is an $F / P L$-bundle over a $Z_{n}$-manifold $M$, we say that $\eta$ may be split if by changing $t$ through a bundle homotopy to $s: E \rightarrow M \times R^{n}$ we have

(i) $s$ is transverse regular to $M \times 0$,

(ii) $s^{-1}(M \times 0) \subset E \rightarrow_{p} M$ is a homotopy equivalence. ${ }^{8}$

The following theorem is essentially due to Novikov.

The Splitting Theorem. Let $\eta=(E, t)$ be an $F / P L$-bundle over $a$ $Z_{n}$-manifold $M$. Suppose that $\operatorname{dim} M \geqq 6, \pi_{1}(M-\delta M)=\pi_{1} \delta M=0$, and that $t$ is a topological homeomorphism. Then $\eta$ may be split.

The splitting obstruction for an $F / P L$-bundle over a $Z_{n}$-manifold is the main tool in studying the obstruction theory described in Theorem 1.

The following surprising theorem is the central result of this analysis.

The Characteristic Variety Theorem. Let $X$ be a finite simplicial complex. Then there is a variety $V$ and $a$ map of $V$ into $X, f: V \rightarrow X$, with the following property: If $\eta=(E, t)$ is any $F / P L$-bundle over $X$, then $\eta$ is trivial iff $f^{*} \eta$ may be split along each component of $V$.

\footnotetext{
${ }^{3}$ If $\operatorname{dim} \delta M=3$, we assume $s^{-1}(\delta M)$ is $P L$-homeomorphic to $\delta M$.
} 
Proof of the Main Theorem. Let $h: L \rightarrow M$ be a topological homeomorphism. Using the obstruction theory we get an $F / P L$-bundle $\eta=(E, t)$ over $L$. Since $h$ is a homeomorphism $t$ can be chosen to be a topological trivialization of $E$. Let $f: V \rightarrow L$ be a characteristic variety of $L$ satisfying the hypotheses of the splitting theorem. Then $f^{*} \eta$ may be split on each component of $V$. The last theorem implies $\eta \sim 0$ and thus $h$ has a triangulation.

The obstruction theory for constructing triangulations of homotopy equivalences is based on framed surgery in the $P L$-category. It is discussed in detail in [4]. The geometrical construction underlying the theory was discovered independently by Wagoner [5]. The Splitting Theorem uses codimension one surgery in the nonsimply connected case (essentially $\pi_{1}=$ free abelian). See [1], [2], and [3]. The Characteristic Variety Theorem makes extensive use of cobordism theory and $\mathrm{K}$-theory. The analysis leads to interesting geometrical interpretations of K-homology and the differentiable Riemann-Roch Theorem. (See Geometric Topology Seminar Notes by D. Sullivan, Princeton University.)

\section{REFERENCES}

1. W. Browder, J. Levine and R. Livesay, Finding a boundary for an open manifold, Amer. J. Math. 87 (1965), 1017-1025.

2. S. P. Novikov, The topological invariance of rational pontrjagin classes, Soviet Math. Dokl. 163 (1965), 298-300.

3. L. Siebenmann, Ph.D. Thesis, Princeton Univ. Press, Princeton, N.J., 1965.

4. D. Sullivan, Triangulating homotopy equivalences, $\mathrm{Ph} . \mathrm{D}$. Thesis 1965 , Princeton University, Rice Univ. (mimeographed).

5. J. Wagoner, Ph.D. thesis, Princeton Univ. Princeton, N.J., 1966.

The University of Warwick, Coventry, England and PrINCETON UNIVERSITY 